\title{
Association of breast milk gamma-linolenic acid with infant anthropometric outcomes in urban, low-income Bangladeshi families: a prospective, birth cohort study
}

\author{
Josyf C. Mychaleckyj $\mathbb{B}^{1,2} \cdot$ Dadong Zhang $^{2} \cdot$ Uma Nayak $^{1,2} \cdot$ E. Ross Colgate ${ }^{3} \cdot$ Marya Carmolli $^{3} \cdot$ Dorothy Dickson $^{3}$. \\ Tahmeed Ahmed ${ }^{4} \cdot$ Masud Alam $^{4} \cdot$ Beth D. Kirkpatrick $^{3} \cdot$ Rashidul Haque $^{4} \cdot$ William A. Petri Jr ${ }^{5,6}$
}

Received: 3 July 2018 / Revised: 17 July 2019 / Accepted: 23 August 2019 / Published online: 9 September 2019

(c) The Author(s) 2019. This article is published with open access

\begin{abstract}
Background/Objectives Infant linear-growth faltering remains a major public health issue in low- and middle-income countries and suboptimal breast milk composition may be a local, population-specific risk factor. The relationship between early post-natal breast milk fatty acid (FA) composition and infant growth at 1 and 2 years of age was investigated prospectively in 563 families in Dhaka, Bangladesh.

Subjects/Methods A maternal breast milk sample drawn before infant age 6 weeks was analyzed for percentage composition of $26 \mathrm{FAs}$, and infant length for age $Z$ score (LAZ) was measured longitudinally to infant age 2 years. Individual FAs were tested as predictors of the infant growth outcomes.

Results Of 26 tested FAs, \%gamma-linolenic acid (\%GLA) was mostly significantly associated with increase in LAZ from 6 to 52 weeks $(\triangle \mathrm{LAZ}(52-6 \mathrm{w}))$, and also to 104 weeks. The association was consistent over all breast milk stages with estimated effect size of $+0.05 \Delta \operatorname{LAZ}(52-6 \mathrm{w})$ per $20 \%$ change in $\%$ GLA $\left(p\right.$ value $\left.=3 \times 10^{-6}\right)$, and stronger for $\Delta \operatorname{LAZ}(104$ $-6 \mathrm{w}$ ) at +0.06 ( $p$ value $=8 \times 10^{-7}$ ), explaining $1 \%$ of the outcome variance. Infant serum zinc measurements at 6 and 18 weeks of age were included in adjusted analyses, suggesting at least partial independence of infant zinc levels. The association was strongest in $417 / 563(74.1 \%)$ families with \%GLA $<0.2 \%$. Breast milk arachidonic acid fraction was within normal range with weaker evidence of association in early breast milk stages.

Conclusions This study found that \%GLA in breast milk was independently associated with infant linear growth, albeit with small effect size, in a predominantly slum-dwelling, low-income, Bangladeshi cohort.
\end{abstract}

Supplementary information The online version of this article (https:// doi.org/10.1038/s41430-019-0498-6) contains supplementary material, which is available to authorized users.

William A. Petri

wap3g@virginia.edu

1 Department of Public Health Sciences, University of Virginia, Charlottesville, VA 22908, USA

2 Center for Public Health Genomics, University of Virginia, Charlottesville, VA 22908, USA

3 Department of Microbiology and Molecular Genetics, Vaccine Testing Center, University of Vermont Larner College of Medicine, Burlington, VT, USA

4 International Center for Diarrhoeal Disease Research, Dhaka, Bangladesh

5 Division of Infectious Diseases and International Health, Department of Medicine, Charlottesville, VA 22908, USA

6 Department of Pathology, University of Virginia, Charlottesville, VA 22908, USA

\section{Introduction}

Breast milk from well-nourished mothers supplies complete nutrition for a neonatal infant, with a composition that adjusts to meet the changing metabolic and nutrient needs of the infant [1]. The critical importance of exclusive breastfeeding to support early infant development is wellestablished and is especially effective in low- and middleincome countries where delay in complementary feeding can reduce the nutritional impact of food insecurity on the infant and direct ingestion of pathogens, while providing passive protection as the nascent infant immune system matures [2, 3]. Although systematic meta-analyses have largely failed to find a significant effect for breastfeeding interventions on post-natal infant anthropometric outcomes [4], the estimated effects are highly heterogeneous, with country income and intervention setting contributing the greatest proportion of heterogeneity [5]. The positive infant 
outcomes derived from exclusive breastfeeding rest on the assumption that expressed milk contains the requisite balance of macro- and micronutrients to sustain the infant at each stage of infant development. Long chain polyunsaturated fatty acids (LCPUFAs) are one class of micronutrient for which there is a strong biological basis for a key role in infant physical growth and cognitive development [6], but maternal or infant supplementation with LCPUFAs has resulted in little significant improvement in post-natal anthropometry outcomes through 1 or 2 years of life [7-9]. This suggests that if deficiencies exist that affect infant growth, they are specific and local.

We previously recruited and followed a cohort of lowincome, mother-infant families in Bangladesh, many living in slums, to study vaccine response and infant growth as components of the PROVIDE Study (Performance of Rotavirus and Oral Poliovirus Vaccines in Developing Countries) [10]. The mothers in the cohorts were given health education, primary care, and encouraged to exclusively breastfeed according to recommended guidelines, but with the high prevalence of micronutrient deficiency in women of reproductive age in Bangladesh [11-13], we were concerned that the mothers may have fed breast milk deficient in micronutrients essential for infant growth. Focusing on the long chain saturated and unsaturated fatty acid (FA) micronutrient composition, we hypothesized that deficiencies in the FA composition of breast milk would be associated with impaired infant linear or ponderal growth [6]. We employed an agnostic analytical approach to look beyond the usual focus on a few key FAs to consider all 26 FAs as equally plausible candidate growth-promoting micronutrients, including intermediate metabolites in the omega- 6 and omega-3 FA pathways.

\section{Methods}

\section{Study population and design}

The PROVIDE study Bangladesh cohort has been reported previously in papers describing the study design [10] and clinical trial outcomes [14, 15]. Further details of Methods are available in Supplementary Materials. Briefly, 700 eligible mothers with their newborns, living in predominantly low socio-economic status households, many in slum conditions in the study recruitment area in Dhaka, Bangladesh, were consented within 7 days post-delivery into the PROVIDE study. The study inclusion/exclusion criteria are listed in Supplementary Table S1. This study was conducted according to the guidelines laid down in the Declaration of Helsinki and all procedures involving human subjects/ patients were approved by the Ethical Review Committee for human subjects protection (protocol \# PR-10060), and
Research Review committee for scientific merit at the International Centre for Diarrhoeal Diseases research, Bangladesh (icddr,b); and by the Institutional Review Boards at the University of Virginia (HSR 15377, sponsor protocol \# CHRMS:M11-112), and University of Vermont (sponsor protocol \# CHRMS:M11-112). Written informed consent was obtained from all subjects.

\section{Procedures}

Infant anthropometry was measured up to 2 years of age, at enrollment and 14 additional visits at weeks $6,10,12,14$, 17, 18, 24, 39, 40, 52, 53, 78, 91, and 104. Trained field research staff measured infant weight to the nearest $10 \mathrm{~g}$ and infant recumbent length to the nearest $0.1 \mathrm{~cm}$ in duplicate. If these were not within $10 \mathrm{~g}$ for weight and $0.1 \mathrm{~cm}$ for length, a third measurement was taken and the average of two measurements within this tolerance was recorded and used to calculate WHO standardized $Z$-scores, length-for-age (LAZ), weight-for-age (WAZ), and weight-for-height (WHZ). Infant gestational age was estimated for a subset of the infants to distinguish fetal growth restriction from prematurity using the Dubowitz-Ballard assessment scale $[16,17]$. Breast milk FA assay procedures have been described previously and are reiterated in Supplementary Methods [18-20]. Individual breast milk fatty acids were expressed as \%wt/wt of total identified FA; the FA profile of each specimen contained 26 individual FAs, listed in Supplementary Table S2. Infant serum zinc $(\mu \mathrm{g} / \mathrm{l})$ was measured using flame atomic absorption spectrophotometry. Serum zinc deficiency was defined as $<640 \mu \mathrm{g} / \mathrm{l}$ [21].

\section{Outcomes}

The primary outcome was the change in infant length-forage $Z$ score (LAZ) from 6 to 52 weeks of age. The secondary outcomes were the change in LAZ from 6 to 104 weeks of age, and corresponding change in WAZ over the same time intervals.

\section{Statistical analyses}

Descriptive statistics of continuous variables were expressed as means \pm standard deviations, and dichotomous variables as proportions. The FA measures were bounded in the composition range $[0,100 \%]$, many were skewed, and the Akaike Information Criterion (AIC) confirmed that the logtransformed variable resulted in a significantly better fit; therefore, all predictor FA composition percentage variables were log-transformed in the statistical models. \%AA and \% DHA were preselected candidates but the other 24 FAs were given equal prior weight as possible predictors of the 
anthropometry outcomes. A two-stage analysis was used: (1) predictor FA variable selection; (2) extensive multiple regression testing. In stage one, the Least Absolute Shrinkage and Selection Operator (LASSO) variable selection method was used to select other non-preselected significantly associated FAs at each step maximizing a penalized likelihood that shrinks nonselected variable coefficients $[22,23]$. The advantage of this method is that a significance test can be applied to each stepwise selected variable that adjusts the degrees of freedom to account for multiple testing of candidate variables. FA variables significant at 0.05 in the LASSO covariance test were carried forward to the second stage of analysis together with preselected \%AA and \%DHA. Detailed discussion of power for FA detection is described in Supplementary Methods and Table S3. In stage two we used multiple linear regression to estimate the effects of the selected FA composition variables after adjustment. The minimally adjusted regression models ('Minimal' model) contained the following preselected variables for adjustment: breast milk arachidonic and docosahexaenoic acid, $\log (\% \mathrm{AA})$ and $\log$ (\%DHA); infant serum zinc at week 6 and week 18; infant age at breast milk sample; infant age at 6 and 18 zinc serum samples; and infant age at week 6 and week 52 (or 104) anthropometry visits. Gestational age was tested as an adjustment in the Minimal model, but where it did not significantly alter an association, the Minimal model results were preferred for increased sample size; estimated gestational age was only available for a subset of the infants. Stratified breast milk stage analyses used the intervals: colostrum, <6 days; transitional, 6-15 days; mature, $>15$ days. Effect sizes and confidence intervals for the logtransformed \%FA predictor variables were presented as untransformed to preserve additivity, or back-transformed to a multiplicative effect size of change in outcome per $20 \%$ change in the \%FA composition, depending on circumstances. All statistical analyses used $\mathrm{R}$ version 3.4. The LASSO analysis used the lars package (https://cran.rproject.org/package=lars).

\section{Results}

\section{Clinical characteristics}

Of 700 families, $563(80 \%)$ provided a breast milk sample that resulted in FA data and were retained in the study with complete infant serum zinc and weeks 6 and 52 anthropometry data, the minimum necessary for primary outcome analyses (CONSORT diagram, Supplementary Fig. S1). The clinical characteristics of mothers and infants are shown in Table 1, at enrollment, study weeks $6,18,52$, and 104. The infants were a mean of 5 days old (range
0-7 days), predominantly born outside the home (74\%) into families subsisting on a mean of approx 140USD per month, and were almost all breastfed at birth (95\%). At 6 weeks, the infants were moderately-to-severely linear growth-impaired (mean WHO length-for-age $Z$ score, LAZ $=-0.96 ; 12 \%$ stunted with $\mathrm{LAZ}<-2$ ) and $22 \%$ were serum zinc deficient, falling to $15 \%$ at week 18 . Figure 1 shows the longitudinal change in LAZ, weight-for-age and weight-for-height $Z$ scores (WAZ, WHZ), at site-specific study visits. The mean LAZ dropped almost inexorably starting immediately after birth, while the WAZ rose to a maximum at approximately week 18, 1 week after the mean end of exclusive breastfeeding at 17 weeks (Table 1), then dropped. These trends were also reflected in the proportion $(28 \%)$ of stunted infants at 52 weeks, rising to $33 \%$ at 104 weeks.

\section{Breast milk composition}

The breast milk specimens were collected during a range of infant ages 3-43 days (mean 10.6), hence 72/563 (12.8\%) were classified as colostrum milk ( $\leq 5$ days), $409 / 563$ (72.6\%) as transitional (6-15 days), and 82/563 (14.6\%) as mature ( $>15$ days). Supplementary Table S2 shows the complete set of 26 FAs assayed, their mean breast milk percentage in all mothers with data $(n=683)$, and in the families with complete outcome and covariate data $(n=$ $563)$. Of note, our total sample mean (SD) values for \%AA and \%DHA were $0.53(0.14)$ and $0.39(0.13)$ respectively.

\section{Selection of fatty acids associated with infant growth}

Running the LASSO procedure for association with the primary outcome weeks ( $\triangle$ LAZ 52-6 weeks) for all 26 FAs (including AA and DHA) resulted in percentage of gammalinolenic acid (\%GLA) being selected at step one with overwhelming association statistical significance despite the in-built correction for multiple FA testing ( $p$ value $=$ 0.00001) (Table 2). The second and subsequent stepwise selected FAs were not significant after correction. Surprisingly, AA was only selected as the fifth-best sequential explanatory FA ( $p$ value $=0.8$ ). These results were also true for $\triangle \mathrm{LAZ}$ (104-6 weeks). The same procedure applied to $\Delta$ WAZ (52-6 weeks) and $\Delta$ WAZ (104-6 weeks) outcomes did not select any FAs at a stepwise $p$ value $<0.05$.

\section{Association of breast milk \%gamma-linoleic acid with infant growth}

Multiple linear regression analyses using the Minimal model, including preselected AA and DHA as possible true associated trophic FAs, confirmed that $\log (\% \mathrm{GLA})$ was the 
Table 1 Clinical characteristics of the PROVIDE infant and mother participants

\begin{tabular}{|c|c|c|c|c|}
\hline \multirow{2}{*}{$\frac{\text { Characteristic }}{\text { Infant enrollment visit ( } 0-1 \text { week })}$} & \multicolumn{3}{|l|}{ Value (SD or \%) } & \multirow{2}{*}{$\begin{array}{l}\text { Value (SD or \%) } \\
n=700\end{array}$} \\
\hline & $n=700$ & \multicolumn{2}{|c|}{ Mother enrollment visit $(0-1 \text { week })^{\mathrm{a}}$} & \\
\hline Enrollment age, days & $5(1.7)$ & \multicolumn{2}{|l|}{ Age, year } & $24.7(4.6)$ \\
\hline Sex (Male), $n(\%)$ & $368(52.8)$ & \multicolumn{2}{|l|}{$\begin{array}{l}\text { Postpartum BMI, } \\
\mathrm{kg} / \mathrm{m}^{2}\end{array}$} & $21.8(3.7)$ \\
\hline Birth order & $1.9(0.8)$ & \multicolumn{2}{|l|}{ Height, $\mathrm{cm}$} & $150.3(5.5)$ \\
\hline Nuclear family only in dwelling, $n(\%)$ & $414(59.1)$ & \multicolumn{2}{|l|}{$\begin{array}{l}\text { First pregnancy, } \\
n(\%)\end{array}$} & $213(30.4)$ \\
\hline Infant gestational age, weeks ${ }^{b}$ & $37.6(1.4)$ & \multicolumn{2}{|l|}{ Education } & \\
\hline Infant gestational age $\leq 36$ weeks, $n(\%)^{\mathrm{b}}$ & $123(32.3)$ & \multicolumn{2}{|l|}{ None, $n(\%)$} & $202(28.9)$ \\
\hline Breastfed at birth, $n(\%)$ & $662(94.6)$ & \multicolumn{2}{|l|}{ Primary, $n(\%)$} & $263(37.8)$ \\
\hline Home birth, $n(\%)$ & $181(25.9)$ & \multicolumn{2}{|l|}{$\begin{array}{l}\text { Secondary or } \\
\text { higher, } n(\%)\end{array}$} & $235(33.6)$ \\
\hline \multirow[t]{2}{*}{ Monthly household expenditure, $1000 \mathrm{~s} \mathrm{Taka}^{\mathrm{c}}$} & $11.5(7.2)$ & \multicolumn{2}{|l|}{$\begin{array}{l}\text { Occupation } \\
\text { homemaker }(\%) \\
n(\%)\end{array}$} & $601(85.9)$ \\
\hline & & \multicolumn{2}{|l|}{$\begin{array}{l}\text { Infant age at } \\
\text { breast milk } \\
\text { sample, days }\end{array}$} & $10.4(6.3)$ \\
\hline Infant visit & 6 weeks $(n=652)$ & 18 weeks $(n=590)$ & 52 weeks $(n=605)$ & 104 weeks $(n=578)$ \\
\hline Age, days & $44.7(2.8)$ & $134.0(5.2)$ & $369.0(6.4)$ & $737.2(6.5)$ \\
\hline Length, $\mathrm{cm}$ & $54.1(1.9)$ & - & $71.4(2.7)$ & $81.7(3.4)$ \\
\hline Weight, $\mathrm{kg}$ & $4.27(0.54)$ & - & $8.2(1.1)$ & $10.1(1.4)$ \\
\hline LAZ & $-0.96(0.93)$ & - & $-1.48(1.02)$ & $-1.61(1.04)$ \\
\hline Stunted LAZ $<-2, n(\%)$ & $81(12.4)$ & - & $167(27.6)$ & $191(33.0)$ \\
\hline WAZ & $-1.01(0.90)$ & - & $-1.18(1.11)$ & $-1.47(1.08)$ \\
\hline Underweight $\mathrm{WAZ}<-2, n(\%)$ & $85(13.0)$ & - & $133(22.0)$ & $174(30.1)$ \\
\hline WHZ & $-0.17(0.98)$ & - & $-0.61(1.04)$ & $-0.73(1.01)$ \\
\hline Wasted WHZ $<-2, n(\%)$ & $14(13.0)$ & - & $45(7.4)$ & $47(8.1)$ \\
\hline$\Delta \mathrm{LAZ}(52 / 104$ weeks -6 weeks $)$ & - & - & $-0.51(0.85)$ & $-0.64(0.95)$ \\
\hline$\Delta$ WAZ $(52 / 104$ weeks -6 weeks $)$ & - & - & $-0.18(0.90)$ & $-0.46(0.99)$ \\
\hline$\Delta$ WHZ (52/104 weeks-6 weeks) & - & - & $-0.46(1.14)$ & $-0.57(1.18)$ \\
\hline Serum zinc, $\mu \mathrm{g} / \mathrm{l}$ & $726(111)$ & $772(147)$ & - & - \\
\hline Zinc deficient (serum zinc $<640 \mu \mathrm{g} / \mathrm{l}), n(\%)$ & $139(22.0)$ & $91(15.4)$ & - & - \\
\hline Serum CRP, mg/l & $1.0(3.7)$ & $3.0(7.7)$ & - & - \\
\hline
\end{tabular}

Continuous data summaries are mean (SD), or categorical $n(\%)$ where indicated; — signifies measures not applicable for this analysis

$B M I$ body mass index, $L A Z$ length for age $Z$ score, WAZ weight for age $Z$ score

${ }^{a}$ Maternal characteristics were collected at infant enrollment (age, postpartum BMI)

${ }^{b}$ Infant gestational age was measured on a subset of 333 infants in Bangladesh to distinguish fetal growth restriction from prematurity using the Dubowitz-Ballard assessment scale

${ }^{\mathrm{c}}$ During the study period 1 USD = approximately 80 Bangladesh Taka

${ }^{\mathrm{d}}$ Range of infant age at breast milk sample was 3-43 days

most significant FA variable (Table $3, p$ value $=3 \times 10^{-6}$, $n=563$ ). The effect size was not different between sexes (interaction test $p$ value $=0.38$ ) and remained significant after adding gestational age to the Minimal model adjustments in a subset of the families $\left(p\right.$ value $=7 \times 10^{-4}, n=$ 318). The transformed adjusted effect size of breast milk \% GLA on mean $\triangle$ LAZ(52-6 weeks) under the Minimal model was $+0.05 \Delta$ LAZ units per $20 \%$ increase in $\%$ GLA
$95 \% \mathrm{CI}[+0.03,+0.07]$ (Table 3 ). The population mean level of \%GLA in breast milk was $0.16 \%(\mathrm{SD}=0.10 \%)$ (Supplementary Table S2). The \%GLA association did not change with continuous sample time point (interaction test of age of infant at breast milk sample and $\log (\%$ GLA $), p$ value $=0.33$ ). To further test for an association with \%GLA resulting from an underlying zinc deficiency in the infants, we reran the models having removed any infant apparently 


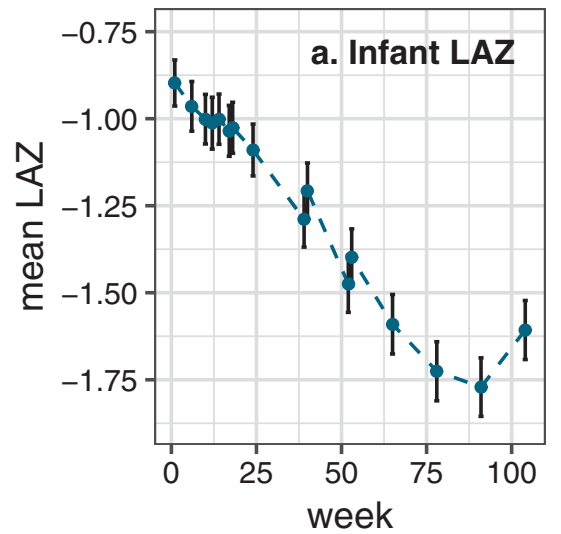

Fig. 1 Plots of the longitudinal change in mean LAZ (a), WAZ (b), and WHZ (c) for infants in the PROVIDE study Bangladeshi cohort. Vertical bars indicate estimated 95\% confidence intervals of the means. Anthropometric data were available to week 104 by protocol
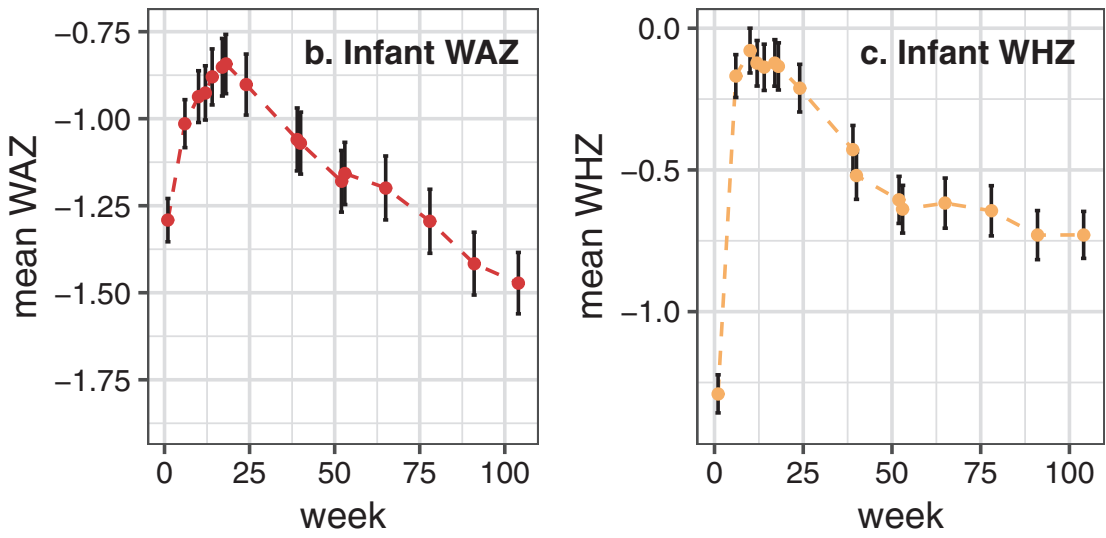

design. LAZ length for age $Z$ score, WAZ weight for age $Z$ score, WHZ weight for height $Z$ score. The $y$-axis ranges for LAZ and WAZ plots are the same but WHZ is dissimilar

Table 2 Results from stepwise selection of breast milk-measured fatty acid composition associated with linear growth faltering outcomes using a penalized selection procedure

\begin{tabular}{|c|c|c|c|}
\hline Outcome & Selection step & $\begin{array}{l}\text { Selected fatty } \\
\text { acid }^{\mathrm{a}}\end{array}$ & $\begin{array}{l}p \text { value for } \\
\text { stepwise selected } \\
\text { fatty acid }\end{array}$ \\
\hline \multicolumn{4}{|l|}{ Primary } \\
\hline \multirow{3}{*}{$\begin{array}{l}\Delta \mathrm{LAZ} \\
\text { (52-6 weeks) }\end{array}$} & 1 & GLA & 0.00001 \\
\hline & 2 & LA & 0.94 \\
\hline & 3 & EDA & 0.6 \\
\hline \multicolumn{4}{|l|}{ Secondary } \\
\hline \multirow{3}{*}{$\begin{array}{l}\Delta \mathrm{LAZ}(104 \\
-6 \text { weeks })\end{array}$} & 1 & GLA & 0.0011 \\
\hline & 2 & LA & 0.59 \\
\hline & 3 & PLA & 0.54 \\
\hline \multirow{3}{*}{$\begin{array}{l}\Delta \text { WAZ (52 } \\
-6 \text { weeks) }\end{array}$} & 1 & GLA & 0.15 \\
\hline & 2 & LA & 0.42 \\
\hline & 3 & PAL & 0.93 \\
\hline \multirow{3}{*}{$\begin{array}{l}\Delta \text { WAZ (104 } \\
-6 \text { weeks) }\end{array}$} & 1 & LA & 0.09 \\
\hline & 2 & AA & 0.25 \\
\hline & 3 & DGLA & 0.88 \\
\hline
\end{tabular}

A stepwise $p$ value of 0.05 was considered significant. Fatty acid variable selection ceased after the first nonsignificant selected fatty acid

$L A Z$ length for age $Z$ score

${ }^{a}$ Fatty acid abbreviations: $A A$ arachidonic acid, $D G L A$ dihomogamma-linolenic acid, $D H A$ docosahexaenoic acid, $E D A$ eicosadienoic acid, GLA gamma-linolenic acid, $L A$ linoleic acid, $P A L$ palmitic acid, $P L A$ palmitolaidic acid

zinc deficient $(<640 \mu \mathrm{g} / \mathrm{l})$ at 6 or 18 weeks of age (Table 1$)$, retaining the zinc levels at 6 and 18 weeks as adjustments in the model. Again $\log (\%$ GLA $)$ retained significance as an independent predictor of $\triangle \mathrm{LAZ}(52-6$ weeks) with effect size $+0.04[+0.01,+0.06], p$ value $=0.0025$. We performed a similar test for maternal zinc deficiency, using breast milk \%
LA/\%DGLA ratio as a proxy [24]. Inclusion of this ratio in the Minimal model still did not eliminate the \%GLA association for $\Delta$ LAZ (52-6 weeks) ( $p$ value $=0.0063$ ). Plots of the primary outcome versus \%GLA revealed the families where reduced \%GLA was associated with the largest length growth deficit (Fig. 2). Mean $\triangle$ LAZ (52-6 weeks) increased with increasing \%GLA at low concentrations of \%GLA $<0.2 \%$, with $0.2 \%$ as a threshold, above which additional increase in \%GLA was not associated with further increase in the outcome. The trends and conclusions remained unchanged after adjusting the primary outcome (Supplementary Fig. S2). There were $417 / 563$ (74\%) of mothers below a \%GLA threshold of $0.2 \%$. Interestingly, the association was stronger for the secondary outcome, $\Delta$ LAZ (104-6 weeks) (Minimal effect size $+0.06[+0.04,+0.09]$, $p$ value $=8 \times 10^{-7}$ ), but less significant for the $\triangle \mathrm{WAZ}$ outcomes (Table 3 ).

\section{No independent association of breast milk \%AA and \%DHA with infant growth}

In the same Minimal model for \%GLA, we saw no independent association of the preselected \%DHA ( $p$ value $=$ 0.69) (Supplementary Table S4), but we did see a nominal association with \%AA ( $p$ value $=0.019)$. However, when we added the estimated infant gestational age at birth to the Minimal model adjustments for the subset of the families with gestational age measured, the association was attenuated to nonsignificance (Supplementary Table S4, $p$ value $=0.071, n=318)$.

\section{Association of \%GLA, \%AA, and \%DHA with infant growth by breast milk stage}

We stratified tests of association under the Minimal model by breast milk stage (Fig. 3 and Supplementary Table S4). 
Table 3 Results for the association of percent gammalinolenic acid in breast milk with linear growth faltering outcomes in the PROVIDE Study

\begin{tabular}{lllll}
\hline Outcome & $n$ & Model $^{\mathrm{a}}$ & $\begin{array}{l}\text { Effect size per 20\% change in \%GLA } \\
{[95 \% \mathrm{CI}]^{\mathrm{b}}}\end{array}$ & $p$ value $^{\mathrm{c}}$ \\
\hline $\begin{array}{c}\text { Primary } \\
\Delta \text { LAZ (52-6 weeks) }\end{array}$ & 563 & Minimal & $0.05[0.03,0.07]$ & $3 \times 10^{-6}$ \\
& 317 & Minimal + GA & $0.05[0.02,0.08]$ & $7 \times 10^{-4}$ \\
Secondary & & & & $8 \times 10^{-7}$ \\
$\Delta$ LAZ (104-6 weeks) & 538 & Minimal & $0.06[0.04,0.09]$ & 0.003 \\
& 298 & Minimal + GA & $0.05[0.02,0.09]$ & $1 \times 10^{-4}$ \\
$\Delta$ WAZ (52-6 weeks) & 563 & Minimal & $0.04[0.02,0.07]$ & 0.002 \\
& 317 & Minimal + GA & $0.05[0.02,0.09]$ & 0.02 \\
$\Delta$ WAZ (104-6 weeks) & 538 & Minimal & $0.03[0.005,0.06]$ & 0.007 \\
\hline & 298 & Minimal + GA & $0.05[0.02,0.09]$ & \\
\hline
\end{tabular}

The primary outcome was the change in LAZ from 6 weeks to 52 weeks

$L A Z$ length for age $Z$ score, $W A Z$ weight for age $Z$ score

${ }^{a}$ Minimal model adjustments: sex, $\log (\% \mathrm{AA}), \log (\% \mathrm{DHA})$, infant serum zinc (week 6), infant serum zinc (week 18), infant age at anthropometry and zinc measures, infant age at breast milk sample Minimal + GA: added gestational age to the Minimal model

b\%GLA in breast milk was tested as $\log$ (\%GLA). Effect sizes and 95\% CI were converted to a standard effect size of the change in outcome $\triangle \mathrm{LAZ}$ or $\triangle \mathrm{WAZ}$ for a $20 \%$ increase in $\%$ GLA. For comparison, one SD increase in \%GLA at the estimated mean \%GLA $(0.16 \%)$ would be a $62.5 \%$ increase in breast milk \%GLA

${ }^{\mathrm{c}}$ The $p$ value tests the significance of the association of $\log (\% \mathrm{GLA})$ with the anthropometry outcome

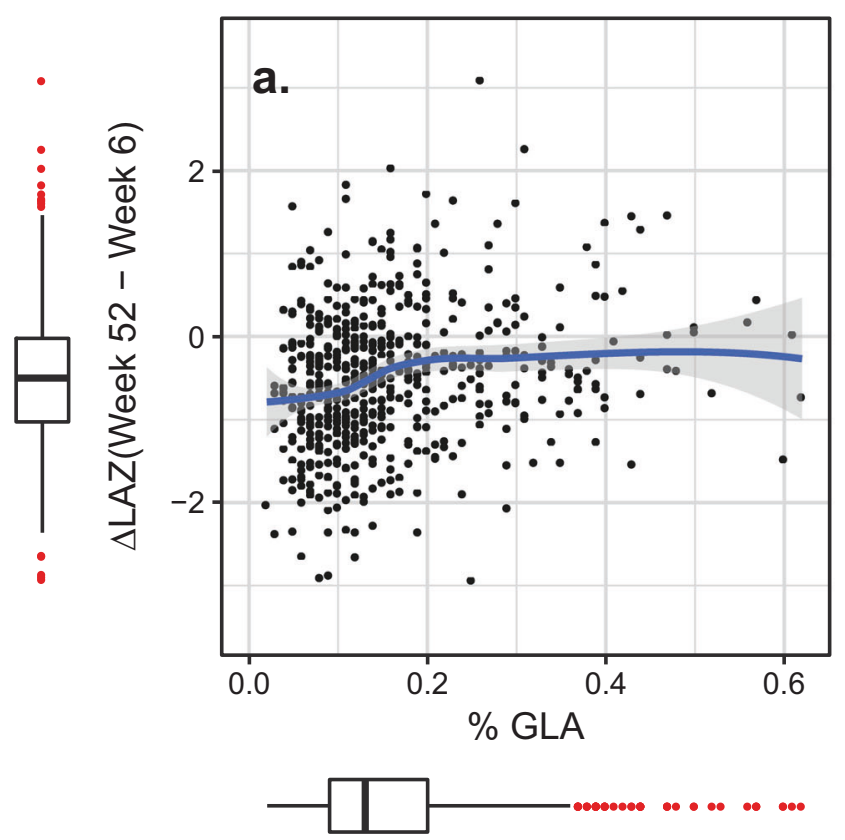

Fig. 2 Plots of the unadjusted primary and secondary outcomes, $\triangle \mathrm{LAZ}$ (week 52-week 6) and $\triangle \mathrm{LAZ}$ (week 104-week 6) against the percentage of gamma-linolenic acid (\%GLA) in breast milk in the PROVIDE study Bangladeshi families. The blue line is the nonlinear local mean at each value of \%GLA fitted by loess algorithm, and the gray envelope is the $95 \%$ confidence envelope. Red points in

The primary association with \%GLA was present in all three stages and largely unchanged in direction or magnitude ( $p$ value trend $=0.61$ ), accounting for the very

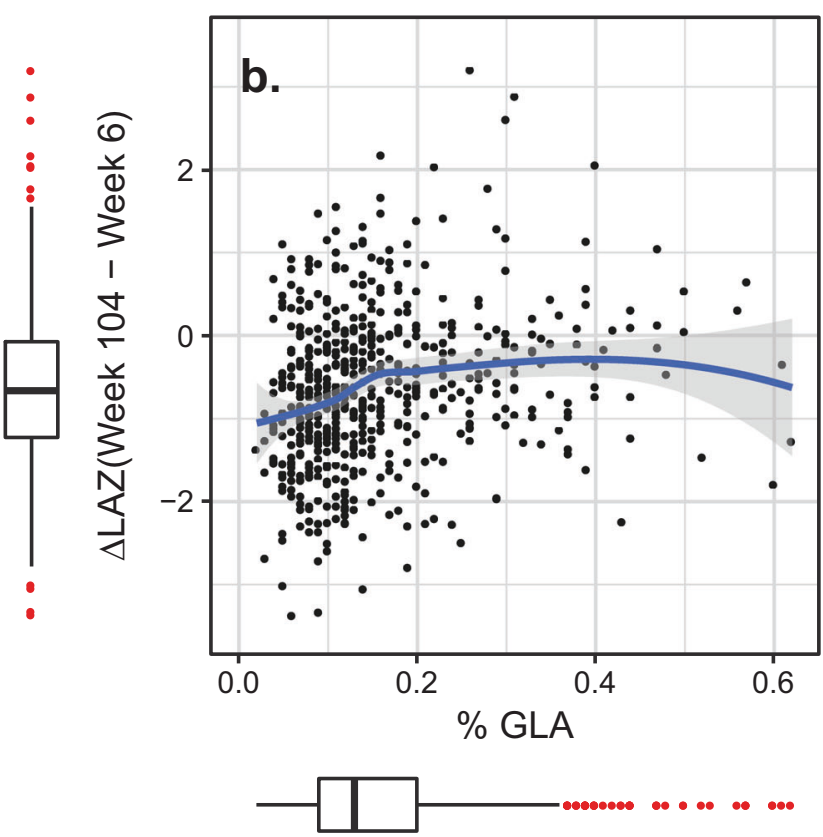

surrounding box plots indicate points outside the range [median$1.5 \times$ interquartile range, median $+1.5 \times$ interquartile range]. LAZ length for age $Z$ score. To magnify the change of interest, the $y$-axis range of the plots omits one extreme outlier infant point at $\triangle \mathrm{LAZ}$ (week $52-$ week 6$)=-5.19, \% \mathrm{GLA}=0.04 ;$ and the same infant $\Delta \mathrm{LAZ}($ week $104-$ week 6$)=-4.49, \% \mathrm{GLA}=0.04$

significant $p$ value for the overall test, including adjustment for continuous age of infant at sample. \%AA was apparently more strongly and significantly positively associated in the 


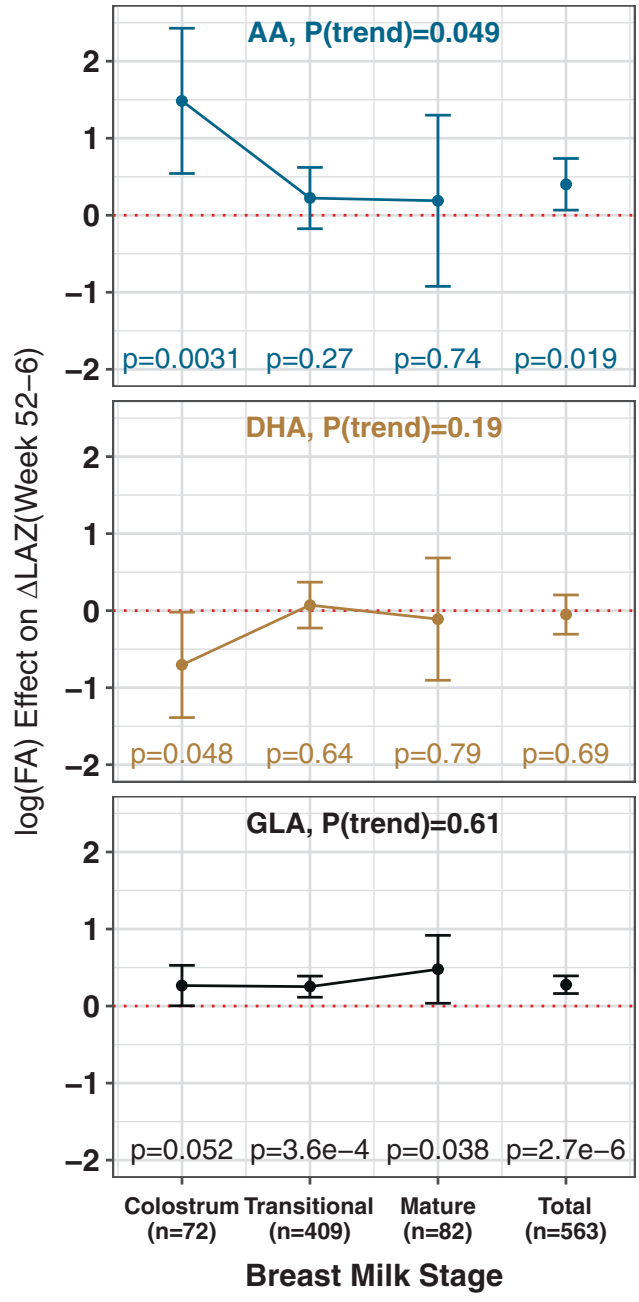

Fig. 3 Plots of the association effect size (beta) for breast milk \%AA, $\% \mathrm{DHA}$, and \%GLA for the primary outcome $\Delta \mathrm{LAZ}$ (week 52-week 6 ), stratified by breast milk stage and sample size $(n)$ for that stage. All three $\log (\% \mathrm{FA})$ were included in each model. Effect sizes are in $\log (\%$ FA) units. Error bars indicate the $95 \% \mathrm{CI}$ range. Individual stage and FA $p$ values are shown for each beta. $P$ (trend) is the $p$ value for a test of trend in the effect sizes from colostrum to mature, $P($ trend $)<0.05$ indicates a significant change in effect size by stage

colostrum stage than \%GLA or \%DHA $(p$ value $=0.0031)$ but was attenuated in transitional and mature stages ( $p$ value trend $=0.049)$; hence, the apparent overall association with $\%$ AA was solely driven by the colostrum stage $(n=72) . \%$ DHA was more weakly and less significantly associated in colostrum stage $(p$ value $=0.048)$ and like \%AA, attenuated to insignificance in the sample-size-reduced gestational ageadjusted model.

\section{Discussion}

The percentage of gamma-linolenic acid (\%GLA) in breast milk sampled up to 6 weeks postpartum was prospectively positively associated with change in infant LAZ from 6 to
52 and to 104 weeks of age in low-income, predominantly slum-dwelling, families in Mirpur, Dhaka, Bangladesh. The association was present at all stages of lactation and was strongest in the $74 \%$ of families where the \%GLA was below $0.2 \%$. The association remained significant after adjusting for possible confounders including infant serum zinc concentration at 6 and 18 weeks of age, and \%AA and $\%$ DHA suggesting that the association of \%GLA is partially or wholly independent of infant serum zinc status and is not a phantom association for \%AA or \%DHA compositional factors. An apparently stronger association of the infant change in LAZ outcome with \%AA limited to the colostrum stage was attenuated to nonsignificance after adjustment with gestational age, and may have represented confounding, but could also have diminished due to reduced sample size. Pervasive secondary associations with other FA members of the omega- 6 pathway in the analyses, seen in the nonsignificant, nonselected second FAs in the LASSO and regression models, suggests that the individual \%GLA association is not an outlier and that there is some relative deficiency in the omega- 6 pathway in this population. The stronger evidence for association of omega- 6 breast milk PUFA variation suggests a greater biological role in infant linear growth than omega-3 PUFAs, including DHA.

Our finding that GLA was the most significantly associated with infant linear growth was unexpected although GLA supplementation can increase infant growth [25]. The positive association with $\triangle \mathrm{LAZ}$ was small in magnitude, with an estimated mean change of +0.05 per $20 \%$ increase in \%GLA for $\triangle \mathrm{LAZ}$ (52-6 weeks) and slightly larger for $\Delta \mathrm{LAZ}$ (104-6weeks), +0.06 . The percentage of variance in the outcome explained by \%GLA was about $1 \%$ for both $\Delta \mathrm{LAZ}$ outcomes.

A plausible a priori explanation for the association was that the \%GLA content was a proxy for a true underlying deficiency in the \%AA breast milk content. Our models all included \%AA or \%DHA as covariate test variables; hence, the residual \%GLA association was independent of their effects and they did not attenuate the association of \%GLA to insignificance. Comparing the mean \%AA and \%DHA in our breast milk samples to published global values [26], our total sample mean(SD) values for \%AA and \%DHA were $0.53(0.14)$ and $0.39(0.13)$ compared to global mean $0.47(0.13)$ and $0.32(0.22)$ respectively (84 studies, $n=2474$ ). For the $27 / 84$ studies with breast milk collected up to 2 months postpartum, our mean values of \%AA and \%DHA ranked above the median value, with multiple high-income country studies showing lower mean \%AA and \%DHA than the PROVIDE families. Hence our means did not appear especially low. Mean \%AA was higher in colostrum than \%DHA but \%GLA was lower than both \%AA and \%DHA in colostrum, so greater \%FA did not consistently result in more significant association with infant growth overall or in a stratified breast milk stage. 
One possible explanation for these results is an underlying pathology of zinc deficiency in a subset of the mothers which could have led to impaired conversion of essential LA to GLA by reduced $\Delta 6$-desaturase efficacy $[27,28]$. We did not measure maternal breast milk or serum zinc levels, but $20 \%$ of the infants were zinc deficient at 6 weeks, and $15 \%$ at 18 weeks, suggesting possible maternal zinc deficiency during the largely exclusive breastfeeding early neonatal period. Since $99 \%$ of AA in breast milk is mobilized from stored maternal depots [29, 30], dysregulation of this enzymatic step would be expected to affect both GLA and AA, although secondary effects of zinc deficiency on FA elongation and $\Delta 5$-desaturation could also affect subsequent enzymatic conversion of GLA to AA. Inclusion of the \%LA/\%DGLA proxy ratio [24] in our models did not eliminate the \%GLA effect. Maternal zinc deficiency remains a possibility, but does not seem to fully explain the association.

Since endogenous omega- 6 and omega- 3 pathways are active in term and preterm neonatal infants [31, 32], the zinc deficiency could extend to infant metabolism. The inclusion of infant serum zinc concentrations at 6 or 18 weeks of age did not eliminate the association with \%GLA, as would be expected if low zinc were the only causative factor. Another possibility is that the association is a marker of the effects of future zinc deficiency, after the infant has transitioned to complementary feeding or has weaned (mean 16 weeks). The nutritional deficiency is likely family-specific, but has less effect in the breastfeeding infant because of the milk supply and prioritization of the mother's reserves to supply the infant. Due to lack of data on plasma zinc levels in the post-breastfeeding infants, we cannot test this hypothesis.

If GLA in breast milk is functionally associated with infant linear growth, there are at least two possible mechanisms for such an effect. If AA is the only true trophic FA metabolite factor in the omega- 6 pathway affecting infant length, then GLA and perhaps other upstream components from AA may represent reservoirs that are diverted to AA synthesis in the infant under conditions of extreme undernourishment. GLA is the most distal upstream omega- 6 after the first rate-limited $\Delta 6$ desaturase step, and hence could be the omega- 6 PUFA of last metabolic resort for future conversion to AA in times of nutritional demand, when the mother is unable to deliver sufficient total volume of preformed growth metabolites in her milk. GLA is only a small fractional component of breast milk, although total infant FA intake depends on total milk intake. Alternatively, GLA may be an independent growth factor, in addition to its role as a precursor for downstream elongation and desaturation. GLA and DGLA are also known to have secondary biological activity [33]. DGLA can be converted into anti-inflammatory eicosanoids with clinical efficacy in treating chronic disease-related processes of inflammation [34]. Supplementation with dietary GLA can directly elevate DGLA, helping to counter the proinflammatory overproduction of eicosanoids from AA [35]. The association of GLA with infant linear growth may not have been previously recognized because prior studies with breast milk and anthropometry data were either underpowered; did not employ a hypothesis-neutral approach for breast milk FA predictors and only focused on AA and DHA; or did not test the association in a population where this specific type of malnutrition is prevalent.

Our study has limitations. We assayed wt/wt percentage of each FA in a single breast milk sample and did not measure the total intake of the FA by the infant. The breast milk composition could be a marker of deficiency both preand post-weaning. This was an observational study with its attendant limitations of causative inference, but we ameliorated this by performing modeling to reduce confounding bias. We cannot definitively rule out other untested confounders, but our analyses suggested that \%GLA was the most significant risk factor, albeit not the FA of largest mean effect size. Between 15 and $20 \%$ of the infants were zinc deficient, and zinc deficiency is likely also contributing to growth deficiency but despite all of our efforts to explain the \%GLA association by zinc levels in the infants, \%GLA remained as an independent associated linear growth factor. Our adjustments for gestational age resulted in a very reduced sample size.

In summary, this study found that \%GLA in breast milk is independently associated with early infant linear growth up 2 years in a low-income, predominantly slum-dwelling, Bangladeshi cohort through all stages of breast milk composition, although the effect size is small. We hypothesize that there is a relative omega- 6 deficiency in this population, from an imbalance in breast milk composition or impaired post-breastfeeding infant metabolism, that affects linear growth. Even after transition to complementary feeding or weaning, the infant is unable to catch up the growth deficit through 2 years of age. Replication is needed to confirm this result and may require specific circumstances of omega- 6 deficiencies, a situation likely uncommon in higher income countries.

Acknowledgements We thank all of the PROVIDE families for their participation and their continued support of the study. We acknowledge the entire study staff in Bangladesh.

Funding This work was supported by the Bill and Melinda Gates Foundation (WAP, grant number OPP1017093).

Author contributions WAP, BDK, RH, TA designed the research; MA, UN, JCM, MC, ERC, DD, conducted the research; UN, DD, DZ, JCM analyzed the data. JCM wrote the paper. WAP had primary responsibility for the final content. All authors read and approved the final manuscript. 


\section{Compliance with ethical standards}

Conflict of interest The authors declare that they have no conflict of interest.

Publisher's note Springer Nature remains neutral with regard to jurisdictional claims in published maps and institutional affiliations.

Open Access This article is licensed under a Creative Commons Attribution 4.0 International License, which permits use, sharing, adaptation, distribution and reproduction in any medium or format, as long as you give appropriate credit to the original author(s) and the source, provide a link to the Creative Commons license, and indicate if changes were made. The images or other third party material in this article are included in the article's Creative Commons license, unless indicated otherwise in a credit line to the material. If material is not included in the article's Creative Commons license and your intended use is not permitted by statutory regulation or exceeds the permitted use, you will need to obtain permission directly from the copyright holder. To view a copy of this license, visit http://creativecommons. org/licenses/by/4.0/.

\section{References}

1. Koletzko B, Rodriguez-Palmero M. Polyunsaturated fatty acids in human milk and their role in early infant development. J Mammary Gland Bio Neop. 1999;4:269-84.

2. Victora CG, Requejo JH, Barros AJ, Berman P, Bhutta Z, Boerma $\mathrm{T}$, et al. Countdown to 2015: a decade of tracking progress for maternal, newborn, and child survival. Lancet. 2016;387:2049-59.

3. Kramer MS, Kakuma R. Optimal duration of exclusive breastfeeding. Cochrane Database Syst Rev. 2012;8:CD003517.

4. Bhutta ZA, Ahmed T, Black RE, Cousens S, Dewey K, Giugliani $\mathrm{E}$, et al. What works? Interventions for maternal and child undernutrition and survival. Lancet. 2008;371:417-40.

5. Giugliani ER, Horta BL, Loret de Mola C, Lisboa BO, Victora CG. Effect of breastfeeding promotion interventions on child growth: a systematic review and meta-analysis. Acta Paediatr. 2015;104:20-9.

6. Yakes EA, Arsenault JE, Munirul Islam M, Hossain MB, Ahmed $\mathrm{T}$, Bruce German J, et al. Intakes and breast-milk concentrations of essential fatty acids are low among Bangladeshi women with 24-48-month-old children. Brit J Nutr. 2011;105:1660-70.

7. Van der Merwe LF, Moore SE, Fulford AJ, Halliday KE, Drammeh S, Young S, et al. Long-chain PUFA supplementation in rural African infants: a randomized controlled trial of effects on gut integrity, growth, and cognitive development. Am J Clin Nutr. 2013;97:45-57.

8. Delgado-Noguera MF, Calvache JA, Bonfill Cosp X, Kotanidou EP, Galli-Tsinopoulou A. Supplementation with long chain polyunsaturated fatty acids (LCPUFA) to breastfeeding mothers for improving child growth and development. Cochrane Database Syst Rev. 2015;7:CD007901.

9. Dewey KG. Reducing stunting by improving maternal, infant and young child nutrition in regions such as South Asia: evidence, challenges and opportunities. Matern Child Nutr. 2016;12:27-38.

10. Kirkpatrick BD, Colgate RE, Mychaleckyj JC, Haque R, Dorothy DM, Carmolli MP, et al. The "Performance of Rotavirus and Oral polio vaccines in Developing countries" (PROVIDE) study: description of methods of an interventional study designed to explore complex biologic problems. Am J Trop Med Hyg. 2015;92:744-51.

11. Arsenault JE, Yakes EA, Islam MM, Hossain MB, Ahmed T, Hotz C, et al. Very low adequacy of micronutrient intakes by young children and women in rural Bangladesh is primarily explained by low food intake and limited diversity. J Nutr. 2013;143:197-203.

12. Shamim AA, Mashreky SR, Ferdous T, Tegenfeldt K, Roy S, Rahman AK, et al. Pregnant women diet quality and its sociodemographic determinants in southwestern Bangladesh. Food Nutr Bull. 2016;37:14-26.

13. Leyvraz M, Laillou A, Rahman S, Ahmed T, Rahman AS, Alam $\mathrm{N}$, et al. An assessment of the potential impact of fortification of staples and condiments on micronutrient intake of young children and women of reproductive age in Bangladesh. Nutrients. 2015;7:9960-71.

14. Mychaleckyj JC, Haque R, Carmolli M, Zhang D, Colgate ER, Nayak U, et al. Effect of substituting IPV for tOPV on immunity to poliovirus in Bangladeshi infants: an open-label randomized controlled trial. Vaccine. 2016;34:358-66.

15. Colgate ER, Haque R, Dickson DM, Carmolli MP, Mychaleckyj JC, Nayak U, et al. A randomized controlled trial with delayed dosing of oral rotavirus vaccine demonstrates decreased risk of rotavirus gastroenteritis associated with serum zinc. Clin Infect Dis. 2016;63:634-41.

16. Dubowitz LM, Dubowitz V, Goldberg C. Clinical assessment of gestational age in the newborn infant. J Pedia. 1970;77:1-10.

17. Ballard JL, Novak KK, Driver M. A simplified score for assessment of fetal maturation of newly born infants. J Pedia. 1979;95:769-74.

18. Nayak U, Kanungo S, Zhang D, Ross Colgate E, Carmolli MP, Dey A, et al. Influence of maternal and socioeconomic factors on breast milk fatty acid composition in urban, low-income families. Matern Child Nutr. 2017;13:e12423

19. Jackson KH, Polreis J, Sanborn L, Chaima D, Harris WS. Analysis of breast milk fatty acid composition using dried milk samples. Int Breast J. 2016;11:1.

20. Harris WS, Pottala JV, Vasan RS, Larson MG, Robins SJ. Changes in erythrocyte membrane trans and marine fatty acids between 1999 and 2006 in older Americans. J Nutr. 2012;142:1297-303.

21. Lin CN, Wilson A, Church BB, Ehman S, Roberts WL, McMillin GA. Pediatric reference intervals for serum copper and zinc. Clin Chim Acta. 2012;413:612-5.

22. Tibshirani R. Regression shrinkage and selection via the lasso. J R Stat Soc B. 1996;58:267-88.

23. Lockhart R, Taylor J, Tibshirani RJ, Tibshirani R. A significance test for the lasso. Ann Stat. 2014;42:413-68.

24. Reed S, Qin X, Ran-Ressler R, Brenna JT, Glahn RP, Tako E. Dietary zinc deficiency affects blood linoleic acid: dihomo-gammalinolenic acid (LA:DGLA) ratio; a sensitive physiological marker of zinc status in vivo (Gallus gallus). Nutrients. 2014;6:1164-80.

25. Fewtrell MS, Abbott RA, Kennedy K, Singhal A, Morley R, Caine E, et al. Randomized, double-blind trial of long-chain polyunsaturated fatty acid supplementation with fish oil and borage oil in preterm infants. J Pedia. 2004;144:471-9.

26. Brenna JT, Varamini B, Jensen RG, Diersen-Schade DA Boettcher JA, Arterburn LM. Docosahexaenoic and arachidonic acid concentrations in human breast milk worldwide. Am J Clin Nutr. 2007:85:1457-64.

27. Clejan S, Castro-Magana M, Collipp PJ, Jonas E, Maddaiah VT. Effects of zinc deficiency and castration on fatty acid composition and desaturation in rats. Lipids. 1982;17:129-35.

28. Huang YS, Cunnane SC, Horrobin DF, Davignon J. Most biological effects of zinc deficiency corrected by gamma-linolenic acid (18: 3 omega 6) but not by linoleic acid (18: 2 omega 6). Atherosclerosis. 1982;41:193-207.

29. Demmelmair H, von Schenck U, Behrendt E, Sauerwald T, Koletzko B. Estimation of arachidonic acid synthesis in full term neonates using natural variation of $13 \mathrm{C}$ content. J Pedia Gastroenterol Nutr. 1995;21:31-6. 
30. Del Prado M, Villalpando S, Elizondo A, Rodriguez M, Demmelmair H, Koletzko B. Contribution of dietary and newly formed arachidonic acid to human milk lipids in women eating a low-fat diet. Am J Clin Nutr. 2001;74:242-7.

31. Salem N, Wegher B, Mena P, Uauy R. Arachidonic and docosahexaenoic acids are biosynthesized from their 18-carbon precursors in human infants. Proc Natl Acad Sci USA. 1996;93:49-54.

32. Szitanyi P, Koletzko B, Mydlilova A, Demmelmair H. Metabolism of 13C-labeled linoleic acid in newborn infants during the first week of life. Pedia Res. 1999;45:669-73.
33. Fan YY, Chapkin RS. Importance of dietary gamma-linolenic acid in human health and nutrition. J Nutr. 1998;128:1411-4.

34. Wang X, Lin H, Gu Y. Multiple roles of dihomo-gamma-linolenic acid against proliferation diseases. Lipids Health Dis. 2012;11:25.

35. Geppert J, Demmelmair H, Hornstra G, Koletzko B. Cosupplementation of healthy women with fish oil and evening primrose oil increases plasma docosahexaenoic acid, gammalinolenic acid and dihomo-gamma-linolenic acid levels without reducing arachidonic acid concentrations. $\mathrm{Br} \mathrm{J}$ Nutr. 2008;99:360-9. 